\title{
A Retrospective Study of the Microbiology of Diabetic Foot Infections at a Community Hospital in Bermuda
}

\author{
Donna Kaye Bennett ${ }^{1}$, Tamar Nasqidashvili ${ }^{1}$, Joseph Saunders ${ }^{1}$, Lavern Swaby ${ }^{2}$, Clyde Wilson $^{2,}$ * \\ ${ }^{1}$ Department of Medicine, King Edward VII Memorial Hospital, Hamilton, Bermuda \\ ${ }^{2}$ Departments of Pathology and Microbiology, King Edward VII Memorial Hospital, Hamilton, Bermuda
}

Email address:

clyde.wilson@bhb.bm (C. Wilson)

${ }^{*}$ Corresponding author

To cite this article:

Donna Kaye Bennett, Tamar Nasqidashvili, Joseph Saunders, Lavern Swaby, Clyde Wilson. A Retrospective Study of the Microbiology of Diabetic Foot Infections at a Community Hospital in Bermuda. International Journal of Diabetes and Endocrinology.

Vol. 6, No. 2, 2021, pp. 76-79. doi: 10.11648/j.ijde.20210602.14

Received: March 29, 2021; Accepted: April 22, 2021; Published: April 30, 2021

\begin{abstract}
There is a high prevalence of type 2 diabetes mellitus in Bermuda. It has been well established that a significant number of patients with diabetes mellitus will develop a diabetic foot infection (DFI), which is associated with increased morbidity and mortality. The objectives of this study were to determine the microbiological and antimicrobial susceptibility profile in DFIs and to investigate whether methicillin resistant Staphylococcus aureus (MRSA) is a common pathogen as well as playing a pivotal role in amputations. A retrospective study was carried out on 96 patients diagnosed with DFI. Demographic data, types of cultures, antimicrobial susceptibility profile and antimicrobial management were collected from medical records. Ninety six patients were investigated and Staphylococcus aureus was the most common organism isolated, followed by Pseudomonas aeruginosa, Group B Streptococcus and Proteus mirabilis. In addition, MRSA organisms were isolated at a low frequency. The organisms isolated showed susceptibility to commonly used antimicrobial agents such as amoxicillin/clavulante, clindamycin, cefazolin, ampicillin/sulbactam, tetracycline, ceftriaxone, trimethoprim-sulfamethoxazole and piperacillin-tazobactam. There was an association between peripheral vascular disease (PVD) and diabetes related amputations. In conclusion, Staphylococcus aureus is a common organism in DFI and the findings of the study does not support empirical MRSA antimicrobial therapy for the management of DFI. There was no evidence to suggest that there is an association between MRSA and diabetes related amputations.
\end{abstract}

Keywords: Diabetes Foot Infection, Amputation, Staphylococcus aureus, Methicillin Resistant Staphylococcus aureus

\section{Introduction}

The prevalence of diabetes mellitus is increasing rapidly in many countries, including the United States of America. A study reported that presently there are an estimated 400 million adults living with diabetes and the global prevalence continues to increase with a projected 500 million people living with the condition by 2035 [1]. The healthcare costs associated with diabetes management are also rising worldwide. Furthermore, it was reported, almost a decade ago, that the direct medical costs associated with diabetes were 176 billion dollars, which is higher than healthcare costs associated with the management of patients with medical conditions not related to diabetes [2]. It has also been reported in the literature, that there is an increasing incidence of diabetes in Bermuda. Moreover, the increasing incidence of diabetes in Bermuda was linked to: family genotype, lifestyle, lack of exercise, obesity and other environmental factors [3]. Diabetes in Bermuda represents a significant burden - with approximately 13,000 people registered as having the condition in 2017 [4]. In addition, the report also mentioned that there was a high limb amputation rate for individuals with diabetes in Bermuda.

A significant number of patients with diabetes will develop DFIs [5]. It has been well established that the bacteria most commonly isolated from DFIs is predominantly Staphylococcus aureus [6]. Furthermore, the prevalence of methicillin resistant Staphylococcus aureus (MRSA) in DFIs has been reported to be $15-30 \%[7,8]$. However, there have 
been reports of a predominance of Gram- negative bacteria DFIs [9]. The development of a DFI is a potential cause of lower limb amputations [10].

This study was undertaken to investigate the bacteria isolated from diabetic patients with lower limb infections and whether the isolation of MRSA had a significant impact on amputations.

\section{Methods}

\subsection{Study Setting}

This retrospective study was undertaken at King Edward VII Memorial Hospital, Hamilton, Bermuda.

\subsection{Study Design}

All patients diagnosed as having a DFI between 2019 and 2020 were investigated in the study. Diabetic foot infection was classified using the Infectious Diseases Society of America's guideline for the diagnosis and treatment of diabetic foot infections [11]. Information regarding microbiology culture and susceptibility testing on isolates obtained from wound swabs, tissue cultures and blood cultures as well as patient demographic characteristics such as age, gender and co-morbid illness, antibiotic therapy, and amputations were identified. All microbiological investigations were performed according to the Clinical and Laboratory Standards Institute's (CLSI) guidelines and local Microbiological guidelines. Identification and susceptibility testing of the isolates were carried out using the Vitek 2 System (bioMerieux, Inc.).

\section{Results}

\subsection{Demographic Characteristics}

A total of 96 cases with the diagnosis of DFI for the period March 2019 to July 2020 were identified and reviewed. The study population compromised of 51 males and 45 females. The age of the patients ranged from 45 to 94 years. Hypertension, dyslipidaemia, peripheral neuropathy and peripheral arterial disease were frequent co-morbidities. The majority of the patients in the study had debridement and $59.3 \%(57 / 96)$ had amputation of a lower limb or toe. $18.7 \%$ $(18 / 96)$ of the lower limb amputations were below the knee, $8.3 \%(8 / 96)$ were above the knee and $41.6 \%$ (40/96) had a digital amputation. The main cause of amputation was peripheral vascular disease (PVD).

\subsection{Microbiological Investigations}

The type of specimens sent to Microbiology for culture and susceptibility testing included wound, tissue, and blood. $60.4 \%(58 / 96)$ of the patients had blood cultures taken for investigation. Only $17.2 \%(10 / 58)$ of blood cultures grew bacteria namely Staphylococcus aureus 3, Group B haemolytic Streptococci 2 and MRSA 2, Morganella morganii 2 and Staphylococcus epidermidis 2. Of the 96 microbiological investigations, 56.2\% (54/96) were culture positive, $66.6 \%(36 / 54)$ were mono-microbial, $33.3 \%(18 / 54)$ were poly-microbial. The predominant organism grown was Staphylococcus aureus $13.5 \% \quad(13 / 96)$ followed by Pseudomons aeruginosa 9.3\% (9/96), Group B Streptococcus 8.3\% (8/96) and Proteus mirabilis 9.3\% (9/96). In addition, methicillin resistant Staphylococcus aureus (MRSA) was isolated from 7 of the patients in the study. Other organisms such as Enterococci, Serratia marcescens, Enterobacer cloaecae, Escherichia coli, Klebsiella pneumoniae, Citrobacter freundii and Morganella morganii are detailed in Table 1. We reviewed the available antimicrobial susceptibility results for the predominant organisms isolated in the study.

\subsection{Antimicrobial Susceptibility Profile}

Nearly one-half of the patients received empirical antimicrobial therapy. Staphylococcus aureus was susceptible to oxacillin, amoxicillin, amoxicillin/clavulanate, cefazolin, gentamicin and clindamycin. The majority of MRSA were susceptible to trimethoprim- sulfamethoxazole, tetracycline, vancomycin, linezolid and gentamicin. Pseudomonas aeruginosa was susceptible to ceftazidime, pipercillintazobactam, gentamicin and meropenem. All of the Group B Streptococcus isolates were susceptible to penicillin. Proteus mirabilis was susceptible to ampicillin/sulbactam, ciprofloxacin, amoxicillin/clavulanate, ceftriaxone, ceftazidime and gentamicin. One Escherichia coli isolate was identified as an extended spectrum beta lactamase (ESBL) producing organism which was susceptible to meropenem and gentamicin. The MRSA isolates were susceptible to vancomycin, linezolid, tetracycline, trimethoprimsulfamethoxazole and gentamicin.

Table 1. The organisms reported from diabetic foot wound and tissue cultures $(n=96)$.

\begin{tabular}{lll}
\hline Organism & Number & Percent (\%) \\
\hline Staphylococcus aureus & 13 & $13.5 \%$ \\
Pseudomonas aeruginosa & 9 & $9.3 \%$ \\
Group B Streptococcus & 8 & $8.3 \%$ \\
Proteus mirabilis & 9 & $9.3 \%$ \\
Methicillin resistant staphylococcus aureus & 7 & $7.2 \%$ \\
Morganella morganii & 3 & $3.1 \%$ \\
Enterobacter cloacae & 3 & $3.1 \%$ \\
Escherichia coli & 2 & $2.0 \%$ \\
Citrobacter freundii & 1 & $1.0 \%$ \\
Enterococcus faecalis & 1 & $1.0 \%$ \\
Klebsiella pneumoniae & 1 & $1.0 \%$ \\
Escherichia coli (extended spectrum beta lactamase positive) & 1 & $1.0 \%$ \\
\hline
\end{tabular}




\section{Discussion}

The objectives of this study were to investigate the microbiological and antimicrobial susceptibility profiles in DFIs as well as to determine whether MRSA is a common pathogen associated with diabetes related amputations. It has been well established that Staphylococcus aureus organisms are most commonly isolated in DFIs [12]. The findings of this study is in agreement with previous reports that Staphylococcus aureus is the predominant organism isolated from DFIs. Other bacteria such as Escherichia coli, Streptococci, Enterococci and Pseudomonas aeruginosa have also been reported to be associated with DFIs [13]. Interestingly, these organisms were isolated at a low frequency in this particular study. It has been reported that the prevalence of MRSA DFIs ranges from $5 \%$ to $20 \%$ [7]. Furthermore, studies from the United Kingdom have reported a high prevalence of MRSA in DFIs [14]. MRSA was isolated in a small number of subjects in this study. However, none of the subjects received empirical MRSA antimicrobial coverage. Our findings do not support empiric use of antimicrobial MRSA therapy for DFI patients. Several studies have identified risk factors for DFIs such as recent antibiotic use, previous hospitalization, extended duration of wound and nasal carriage of MRSA $[15,16]$. Some of the patients in the study who had a wound or tissue culture positive for MRSA, were also previously colonized with the organism. This observation is compatible with reports which identified colonization as a risk factor for MRSA DFIs. A recent study reported high level antimicrobial resistance among Gram-negative organisms isolated from DFIs [12]. However, only one Gram-negative organism, namely an extended spectrum beta lactamase (ESBL) was identified in this study.

The most common empiric antimicrobial agents used for management of patients presenting with DFIs were ampicillin/sublactam, amoxicillin/clavulanate, clindamycin, ciprofloxacin and piperacillin/tazobactam. In addition, the data showed that the antimicrobial agents that were used for empirical management provided coverage for the most predominant organisms Staphylococcus aureus and Pseudomonas aeruginosa. Furthermore, empiric antimicrobial therapy was frequently modified according to the culture and susceptibility results. A process improvement initiative has been introduced and empiric antimicrobial therapy guidelines are currently available online on the hospital's Intranet. This initiative highlights good antimicrobial stewardship and the need to use antimicrobial agents judiciously. The Infectious Diseases Society of America (IDSA) guidelines for the treatment of DFIs recommends that a broad spectrum of antimicrobial agents should be used for the treatment of DFIs [11]. Most of the patients in the study had debridement and some form of amputation. It has been reported that amputation rates in diabetic patients is an indicator of a health system's performance regarding the management of the condition. Furthermore, reducing the incidence of amputations amongst diabetic patients indicates a more effective use of the healthcare spectrum of diabetes and related complications $[17$, $18]$.

\section{Conclusion}

In conclusion, Staphylococcus aureus was the most frequently isolated organism in this study. The majority of the positive cultures were mono-microbial and MRSA organisms were isolated at a low frequency. Antimicrobial resistance is a common observation in DFIs. The absence of significant antimicrobial resistance in the study suggests good compliance with infection prevention and control policies and the judicious use of antimicrobial agents. Interestingly, there was no significant correlation between the various types of organisms isolated, PVD and amputation. Further studies exploring molecular methodology are required to identify pathogens responsible for DFIs. It is important to monitor the prevalence of MRSA to facilitate clinical decision making in the management of DFIs and in identifying patients at high risk. The introduction of a multidisciplinary team approach using care pathways and education will decrease the incidence of diabetes related lower limb amputations.

\section{Conflict of Interests}

The authors declare that they have no competing interests.

\section{References}

[1] Centres for Disease Control and Prevention, National Diabetes Statistics Report, 2020. Atlanta, GA: Centres for Disease Control and Prevention, US. Dept. of Health and Human Services; 2020: Available at http//www.cdc.gov/diabetes/pubs/statsreport14/nationaldiabetes-report-web.pdf: 2020.

[2] Guariguata I, Whitting DR, Hambleton I, Beagley J, Linnenkamp U, Shaw JE. Global estimates of diabetes prevalence for 2013 and projections for 2035. Diabetes Res Clin Pract. 2014; 103: 137-149.

[3] Parris D, The alarming increase of diabetes in Bermuda. West Indian Med J 2014; 63: 685.

[4] Dialysis in Bermuda, Bermuda Health Council. 2018. http://bhec.bm/wp-content/uploads/2018/041/issue-briefdialysis-v1-20180416.

[5] Kwon KT, Armstrong DG. Microbiology and antimicrobial therapy for diabetic foot infections. Infect Chemother. 2018; 50: 11 .

[6] Lavery LA, Fontaine JL, Bhavan K, Kim PJ, Williams JR, Hunt NA. Risk factors for methicillin resistant Staphylococcus aureus in diabetic foot infections. Diabet Foot Ankle 2014; 5: 23575.

[7] Eleftheriadou I, Tentolouris N, Argiana V, Jude E, Boulton AJ. Methicillin resistant Staphylococcus aureus in diabetic foot infections. Drugs 2010; 70: 1785-97. 
[8] Stacey HJ, Clements CS, Welburn SC, Jones JD. The prevalence of methicillin resistant Staphylococcus aureus among diabetic patients: a meta-analysis. Acta Diabetol. 2019; 56: $907-21$

[9] Shankar E, Mohan V, Premalatha R, Srinivasan S, Usha A. Bacterial aetiology of diabetic foot infections in South India. Eur J Int Med 2005; 16: 567-70.

[10] Consensus Development Conference on diabetic wound care. Diabetes Care. 1999; 22: 1354-60.

[11] Lipsky BA, Berendt AR, Comia PB, Pile JC, Peters EJ, Armstrong DG, et al. 2012 infectious diseases society of America clinical practice guideline for the diagnosis and treatment of diabetic foot infections. Clin Infect Dis. 2012; 54: 1679-84.

[12] Mutonga DM, Mureithi MW, Ngugi NN, Otieno FCF. Bacterial isolation and antibiotic susceptibility from diabetic foot ulcers in Kenya using microbiological tests and comparison with RT-PCR in detection of S. aureus and MRSA. BMC Res Notes 2019; 12: 244 Doi.org/10.1186/s13104-0194278-0.

[13] Reveles KR, Duhon BM, Moore RJ, Hand EO, Howell CK. Epidemiology of methicillin -resistant Staphylococcus aureus diabetic foot infections in a large academic hospital: Implications for antimicrobial stewardship. PLOS ONE. 2016; 11: 11 doi: 10.1371/journal.pone.0161658.

[14] Dang CN, Prasad YDM, Boulton AJM, Jude EB. Methicillin resistant Staphylococcus aureus in the diabetic foot clinic: a worsening problem. Diabet Med. 2003; 20: 159-161.

[15] Richard JL, Sotto A, Jourdan N et al. Risk factors and healing impact of multidrug-resistant bacteria in diabetic foot ulcers. Diabetes Metab. 2008; 34: 363-369.

[16] Haleem A, Schultz JS, Heilmann KP et al. Concordance of nasal and diabetic foot ulcer Staphylococcal colonization. Diagn Microbiol Infect Dis. 2014; 79: 85-89.

[17] Jeffcoate WJ, Margolis DJ. Incidence of major amputation for diabetes in Scotland sets a target for us all. Diabetes Care. 2012; 35: 2419-20.

[18] Kennon B, Leese GP, Cochrane L, Colhous H, Wild S, Stang $\mathrm{D}$ et al. Reduced incidence of lower extremity amputations in people with diabetes in Scotland: A nationwide study. Diabetes Care. 2012; 35: 2588-90. 